REVISTA CHILENA DE LITERATURA

Diciembre 2016, Número 94, 15-38

\title{
LA LECTURA EN EL ENTORNO DIGITAL: NUEVAS MATERIALIDADES Y PRÁCTICAS DISCURSIVAS
}

\author{
José Antonio Cordón \\ Universidad de Salamanca \\ jcordon@usal.es
}

RESUMEN / ABSTRACT

La lectura ha experimentado numerosas transformaciones a lo largo de la historia configurando una serie de cambios que se han trasladado a las prácticas y discursos sobre la misma. La aparición de los contenidos digitales y la generación de un ecosistema en torno a estos ha generado profundas transformaciones en una tradición de siglos, arbitrando nuevos modelos de producción y recepción en los que la práctica individual, ensimismada y concentrada se complementa con sistemas algorítmicos en los que lo sustancial recae en los modelos subyacentes en miles de obras, más que las particularidades de una sola. Socialización y editorialización de la web representan dos de las principales tendencias de la lectura digital.

Palabras clave: lectura digital, distant reading, lectura social, editorialización digital.

The practice of reading has undergone numerous transformations throughout history which has resulted in a series of changes in the practices and discourses about it. The emergence of digital contents and the creation of an ecosystem around them has generated profound transformations in a tradition of centuries, making available new models of production and reception in which the individual, self-absorbed and concentrated practice is complemented with algorithmic systems in which the essential relies on the underlying models of thousands of works, rather than on one in particular. Socialization and editorializing of the web represent two of the major trends in digital reading.

KEYWORDS: digital reading, distant reading, social reading, digital editorializing. 


\section{INTRODUCCIÓN}

Libros y lectura han guardado una curiosa relación a lo largo de la historia $\mathrm{y}$, singularmente, desde la invención de la imprenta: mientras los libros han ido creciendo en progresión geométrica, la lectura lo ha hecho en progresión aritmética, describiendo una relación que en muchos casos se ha convertido, paradójicamente, en inversamente proporcional, generando una de las grandes contradicciones de nuestro tiempo. El crecimiento exponencial de la publicación de obras no ha ido acompañado de un movimiento similar en la lectura, o en la compra de las mismas. Si la lectura y el pensamiento crítico que acompaña a la misma constituye uno de los signos de la modernidad, la retracción que, aparentemente, está experimentando ésta representaría otro de los síntomas de la desintegración de una fenomenología bibliocéntrica fagocitada por la aparición de medios de expresión más eficaces desde el punto de vista comunicativo.

Además, el ecosistema tradicional del libro y de la lectura se ha visto alterado por la aparición de tecnologías de carácter disruptivo que, por primera vez en la historia de la edición, afectan a toda la cadena de valor del libro, desde la autoría a la recepción, articulando un sistema nuevo, emergente, que ya está produciendo desplazamientos significativos, cuando no migraciones irreversibles, entre los soportes impresos y digitales. Las tensiones y conflictos derivados de esta dialéctica se han sustanciado de diferentes modos, generando movimiento de resistencia, bien por la vía del rechazo, bien por la de la asimilación, como la representada por el llamado "skeuomorphism", esto es la práctica de incorporar elementos obsoletos en un diseño, mantenidos por familiaridad o por tradición, a pesar de que ya no sirven a ningún propósito funcional. Un ejemplo de ello lo constituye el libro digital, que aún reviste un claro carácter mimético respecto a la obra impresa que, en muchos casos, toma como referente estético y conceptual (Sinclair; Rockwell 2014).

Que la lectura constituye una de esas raras prácticas que concitan la unanimidad en cuanto a su consideración positiva, es algo que nadie pone en duda, incluso en los momentos actuales en los que los discursos de legitimación se han ido diversificando con ponderaciones más distribuidas en torno a otros discursos culturales. La lectura, tomada genéricamente, ha gozado del beneplácito social, al menos desde las postrimerías del siglo XIX, cuando los movimientos en favor de la alfabetización, como medio de integración social, y de promoción y acceso a los bienes culturales, pasaron a constituirse 
en objetivos estratégicos de las políticas estatales. Esto no fue óbice para que existieran movimientos de control respecto a determinados tipos de lecturas, motivados por sectores religiosos, políticos o sociales. Pero incluso estos pronunciamientos, plasmados en diferentes formas de censura, no hacían sino conferirle un estatus especial, aunque fuera por la vía de la negación.

Saber leer se ha considerado como un bien en sí mismo y la incorporación de su aprendizaje a los programas formativos de todos los países del mundo evidencia el grado de consolidación de esta convención.

Sin embargo, su evolución y desarrollo están sujetas a múltiples interpretaciones sobre la viabilidad de las estrategias seguidas o sobre la oportunidad de las mismas (Arguelles, 2009).

Las cifras que arrojan los diferentes estudios e informes que se publican en todo el mundo representan igualmente un elevado grado de inconcreción, por lo que es difícil saber cuánto se lee y, sobre todo, cómo se lee. Todos los países han incorporado la cuantificación de lo digital en sus anuarios, panorámicas y observatorios, pero las cifras que arrojan revisten un carácter siempre aproximativo, pues la irrupción de la autopublicación como uno de los escenarios más potentes del ecosistema digital dificulta la normalización de la práctica estadística en este terreno. De tal manera que la industria editorial de cada país, o los organismos encargados de su estudio, dan fe de las prácticas de publicación que podíamos considerar como "regladas", regidas por estándares internacionales y sujetas a sistemas de control bibliográfico que garantizan la fiabilidad de las cifras, pero queda un extenso margen, cada vez menos periférico, que es preciso tener presente cuando se contempla esta nueva realidad. Por ejemplo, en la última estadística sobre los libros electrónicos publicada en España (Panorámica 2016), se informa de que la producción de estos muestra, en 2015, un incremento del 2,9\%, con un total de 20.848 ISBN (20.256 ISBN en 2014), representando el 26,3\% del total de la producción, pero no se aporta ningún dato sobre las dimensiones de la autopublicación, cuyas cifras incrementarían considerablemente los números anteriores. De hecho, las cifras aportadas por el Comercio Interior del Libro, el informe que anualmente elabora el Gremio de Editores de España, para el año 2015, difieren significativamente de las aportadas por la Panorámica de la Edición Española de libros, elaborada por el Ministerio de Cultura. Según el Comercio Interior (2016), los libros electrónicos publicados en 2015 fueron 44.209, más del doble que los asignados para ese año por la otra fuente comentada. La diferencia entre unas cifras y otras está sin duda en la autopublicación. 
De cualquier modo, los estudios se han incrementado desde el momento en el que lo digital interviene como un elemento más o menos disruptivo en las prácticas desarrolladas por los lectores de todos los países del mundo, trazando un panorama que nos permite avizorar escenarios contextualizados sobre la forma en que circulan y se consumen los contenidos digitales.

\section{DE LA LECTURA INDIVIDUAL A LA LECTURA COLABORATIVA}

Uno de los fenómenos más interesantes acaecidos en los últimos años ha sido el cambio que la condición del lector ha experimentado en el contexto literario en el que los diferentes estudios y conceptualizaciones, como los deudores de la noción de "campo editorial" (Bourdieu 2011), lo confinaban a un estatus finalista en el que la recepción constituía su ubicación singularizada. A lo largo de la historia el lector ha ocupado un papel pasivo, de mero receptor, sin más intervención en el proceso de publicación que las opiniones que, ocasionalmente, podía formular a la editorial o al autor a través de sistemas convencionales como presentaciones de libros, reuniones ad hoc para valorar algunas colecciones, o mediante los buzones de sugerencias o mail arbitrados por algunas editoriales para facilitar estos procesos comunicativos. La mayoría de los textos canónicos sobre edición, las memorias de editores y autores, los testimonios alentados por iniciativas de todo tipo, han arbitrado un protagonismo tácitamente pasivo, en el que el destinatario de la escritura se situaba al otro lado de esta y, en todo caso, en uno distinto del ocupado por la creación.

En este contexto, el lector ha sido objeto de numerosos estudios en los que, cuando se ha valorado su actividad, se ha hecho como intérprete de la recepción, en figuras como las del lector implícito o lector real, que desarrollara Iser (2008), para quien un texto literario solo puede desarrollar su efecto cuando es leído. Incluso cuando plantea que es el lector el que crea y reconstruye el texto, afirmando que la teoría de la recepción "siempre tiene que ver con lectores que se constituyen históricamente", se refiere a lectores que no intervienen, que no pueden intervenir en el circuito primario de la generación de contenidos. Esto no quiere decir que el lector haya permanecido ajeno al texto o pasivo ante el mismo, pues la lectura es una de las actividades más reactivas que existen. Pocos lectores, independientemente del género de que se trate, han permanecido nunca impasibles ante el mismo. Comentarios, recomendaciones, críticas y matizaciones han acompañado siempre a las 
publicaciones, dando lugar a intervenciones más o menos especializadas cuyo ejemplo más formalizado lo han constituido los marginalia que, en forma de subrayados, llamadas y acotaciones aparecen a lo largo de toda la historia de los textos impresos, constituyendo un material de primera mano para el desarrollo de todo tipo de estudios culturales, como lo demostrara Peter Burke (2009), entre otros, con su magnífica interpretación de El Cortesano a la luz de las mismas. O bien el uso de las citas como elemento de intertextualidad y comunicación entre las obras y los autores (Finnegan 2013).

De cualquier modo, el libro se convertía en una suerte de palimpsesto interpretable únicamente por su manipulador, que controlaba las claves del proceso, siempre que la consulta no difiriera demasiado tiempo respecto a su intervención.

Sin embargo, las intervenciones de los lectores tenían, en su mayoría, un carácter cerrado e intransitivo $\mathrm{y}$, en todo caso, se trataba de un discurso dominado, en sus elementos paratextuales, por el editor o el autor que marcaba los factores periféricos de la obra que podían señalizarse: cuartas de cubierta, fajas, solapas, así como los elementos estructurales inherentes a ella: tripa de los libros: estilo, fuentes, párrafos, índices, etc.

Esta jerarquía de los elementos paratextuales y estructurales, durante siglos supervisada por editores y autores, está experimentando un desplazamiento hacia el lector, de tal manera que el mismo asume un protagonismo que afecta tanto a los textos como a la comunicación de los mismos. El lector social ha propiciado la aparición de la lectura social, que adquiere consistencia y significación en el contexto digital (Cordón, Gómez, Arévalo 2013).

En este sentido, uno de los aspectos más significativos es la transformación que el uso de las redes sociales ha introducido en las formas de leer. La necesidad que tienen los autores de competir por un espacio cada vez más concurrido ha provocado que sus intervenciones, a través de los escenarios en los que se desenvuelven los lectores, sean cada vez más intensas. Esto ha suscitado la ruptura de la separación tradicional entre los circuitos de producción y recepción. La función del autor no finaliza cuando la obra está en el mercado, como ocurría en el circuito impreso, sino que prosigue una vez se ha lanzado para su consumo, o establece una línea de continuidad en todo el proceso, gracias a la cual los lectores reciben información permanente sobre el desarrollo del mismo. La intervención en redes como Twitter, Facebook, Goodreads, LibraryThings y similares constituye una caja de resonancia imprescindible para conferirle a las obras una visibilidad y proyección inevitable en un contexto cada vez más competitivo. Joyce Carol Oates, por ejemplo, se ha 
labrado una sólida reputación en Twitter, no solo porque comenta a través de esta red todo tipo de noticias, sino, sobre todo, porque da pistas sobre sus obras, difíciles de encontrar por otra vía. Escritores como Rowling o George RR Martin cuentan con club de fans que reciben a través de Twitter detalles sobre el desarrollo de sus obras, compartiendo informaciones que no figuran en las mismas y que sirven para esclarecer algunos de sus desarrollos. Esta reducción de la distancia existente entre los dos polos del circuito, autor y lector, ha modificado en muchos casos el proceso creativo, en tanto que el autor conoce las inquietudes, necesidades o aspiraciones de su público natural y puede adaptarse a él, al igual que el proceso de lectura en tanto que esta no constituye un hecho aislado e intransitivo en el que la interpretación reviste un carácter autónomo, sino que se inscribe en un entorno de relaciones y conversaciones, en las que el autor y otros lectores protagonizan y colaboran los sistemas de decodificación.

El último informe de Smartword sobre la edición independiente y la autopublicación muestra la influencia que las redes sociales revisten en el incremento de las ventas. Los autores mejor retribuidos cuentan con sitios web particulares e intervienen regularmente en las redes.

\section{Bestselling Authors More Likely to Have a Website}

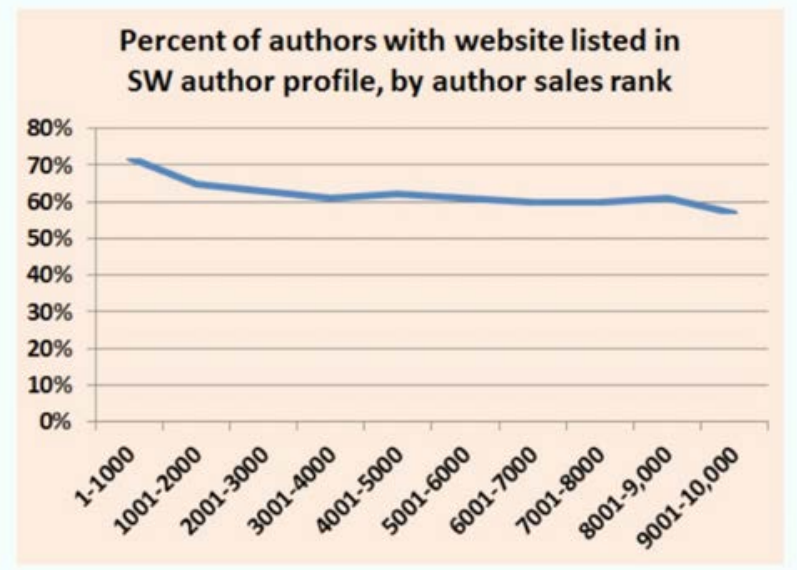

Fig. 1. Annual Smashwords Survey 2015

http://www.slideshare.net/Smashwords/2015-smashwords-survey-how-to-sellmore-ebooks 
De lo que se trata, como se señala en el informe, es de promover buenas prácticas entendidas en un contexto social. Se definen estas como aquellas que proporcionan visibilidad, accesibilidad, compromiso del lector y entretenimiento a las obras.

Algunos de los editores científicos más destacados en la actualidad, así como reputados investigadores, inciden en la importancia de las redes sociales para una adecuada difusión de los trabajos de investigación, postulando la necesaria integración de las mismas en la hoja de ruta digital de cualquier editorial o proyecto de investigación (Michael 2016).

La industria editorial va incorporando estas prácticas a través de colecciones y productos que recogen esta filosofía del intercambio y la comunicación. Paragraph y Atria Books, filiales de Simon and Shuster, lanzaron, en diciembre de 2015, su aplicación Crave. La nueva propuesta editorial se centra en uno de los segmentos de mercado más importantes en la venta de contenidos digitales: la novela romántica que, a través de esta aplicación intenta reinventar su consumo. Los abonados reciben cada día un pasaje de la obra deseada, junto con videos y fotos de los personajes principales, con la particularidad de favorecer la interacción con ellos. Los personajes principales de las obras envían mensajes a los lectores, complementando con anécdotas e informaciones complementarias el perfil de los mismos. Se persigue de esta manera incrementar los rasgos inmersivos de la historia confiriéndole continuidad a la misma mediante intervenciones externas a la obra. El texto se transforma en un elemento más de un conjunto narrativo en el que los elementos que intervienen en el mismo, autor, personajes, lectores, contribuyen a la reinterpretación del contenido.

La consideración del libro como algo sólido, inmutable y permanente se transforma dando lugar a una percepción más fluida, sujeta a cambios tanto en el tono como en el contenido por la intervención de diferentes actores en la conversación.

La apertura de diferentes espacios de conversación da lugar a lo que podíamos denominar como lectura ecléctica, esto es, aquella que no solo discurre a través de formas variadas de intervención, sino que transita por soportes diferentes sin solución de continuidad. Las transiciones entre lo impreso y lo digital son cada vez más abundantes como han demostrado las numerosas ofertas de sistemas mixtos en el ámbito editorial. Son ya muchas las editoriales que trabajan simultáneamente este doble entorno, las que ofrecen obras digitales con la compra de los libros impresos, o al contrario las que, habitualmente mediante edición bajo demanda, permiten 
la impresión de una obra que se ha editado previamente en digital. Y van surgiendo soluciones imaginativas como la que representa Shelfie (http:// www.shelfie.com/), una aplicación lanzada por una Start Up de Vancouver, que permite, a partir de una foto de una estantería con libros físicos, recibir los mismos en formato digital.

Lectura ecléctica facilitada por la progresiva interoperabilidad que caracteriza a las textualidades digitales, navegables a través de todo tipo de pantallas. Los contenidos se van convirtiendo en zonas de inmersión a través de todo tipo de dispositivos, independientemente de la ubicación y naturaleza de estos.

En este sentido, el autor ha ido abandonando las prácticas propias de la edición impresa para sumergirse en experiencias que aprovechan las ventajas y posibilidades del entorno digital, tanto en lo relativo a sus interacciones con los lectores, como en lo referente a la concepción de la propia obra, que se articula como un sistema de intervenciones múltiples. Iain Pears, con motivo de la aparición de su última obra, en agosto de 2015, comentaba que había alcanzado los límites de la narración en formato impreso, viéndose obligado a explorar nuevas vías. Esa ha sido su ocupación durante los últimos cinco años, con la elaboración de su obra Arcadia, lanzada primero en digital en el mes de agosto de 2015 y en papel en el mes de septiembre, aunque esta última no es tan completa como la versión digital. De hecho, el libro impreso cuenta con 180.000 palabras, mientras que en el digital hay 250.000. Además, según señala el autor, para comprender adecuadamente la obra se hace imprescindible el uso de la aplicación, pues la obra está concebida para su recorrido digital. 


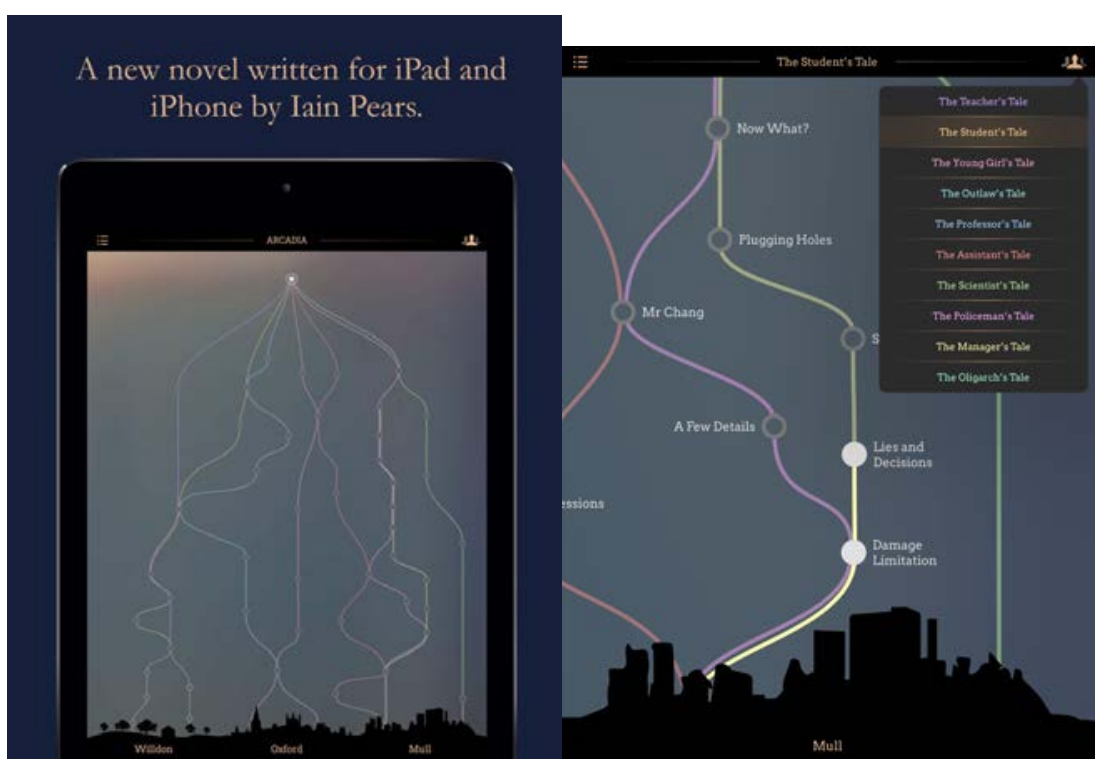

Fig. 2. Arcadia de Iain Pears

En esta obra se presentan 10 relatos diferentes correspondientes a otros tantos personajes. Según las elecciones del lector la historia evoluciona de diferente manera convirtiéndose, parafraseando a Juan Goytisolo (quien a su vez lo hacía de Borges), en una suerte de Jardín de los textos que se bifurcan.

Esta iniciativa, y muchas otras similares responden a la filosofía de "Digital First". Esta consiste en el lanzamiento de la versión digital primero, con todas sus prestaciones asociadas, y la posibilidad de la publicación impresa como un producto derivado y secundario. Se trata de una evidencia más de la transformación que se está produciendo en el sistema de prioridades del sector editorial.

La cuestión que se plantea con estos desarrollos es la existencia de realidades separadas para los mismos productos cuando se trata de circuitos diferentes. Empieza a ocurrir con los libros lo que ya se había comenzado a experimentar en el ámbito de las revistas, cuando se produjeron las primeras fases de la migración de los soportes impresos a los digitales, esto es, que el gradiente de las funcionalidades iba basculando progresivamente hacia lo digital en detrimento de lo físico, hasta que, llegado un punto, se abandonaba completamente el soporte de referencia. En la lectura digital se han ido desarrollando etapas en las que la propensión imitativa de lo impreso ha ido 
convirtiéndose en la articulación de un entorno diferenciado y cada vez más autónomo, representado por obras en las que las versiones digitales e impresas empiezan a distanciarse considerablemente, ofreciendo experiencias de lectura netamente discriminadas. En este sentido, la relación impreso-digital se podría inscribir en el ámbito de la dialéctica, en cuanto a lucha de los opuestos y, más que de una polarización, podríamos hablar de una tensión productiva entre ambas en tanto que se produce una retroalimentación permanente. La necesidad de prevalecer y competir por un mercado similar obliga a una mejora intensiva del producto en sus conceptos clásicos, en el entorno impreso, y a una innovación regular de las funcionalidades en el entorno digital. El libro impreso ha llegado a un punto de clausura fundamental en el que sus desarrollos recaen en propuestas redundantes de un solipsismo profesional cuya única meta es la reiteración perfeccionista de sus elementos constitutivos. La condición de posibilidad inherente a cualquier invención ha desaparecido en beneficio de una progresiva normalización cuya finalidad es únicamente afianzar los logros establecidos, conservadora en sus propósitos y en sus actitudes. Lo impreso, por conservador lleva implícito el concepto de fidelidad, frente a la infidelidad propia de lo digital, siempre atento a los cambios y trasformaciones, en las que los desplazamientos pueden ser constantes. Frente al formalismo sólido del entorno impreso lo digital se mueve en una deriva necesariamente provisoria, en que el tópico de la finitud vinculado con la obsolescencia puede estar inextricablemente vinculado con el fracaso que, en lugar de auspiciar renuncias y abandonos, suele promover nuevos proyectos e iniciativas. Esta retroalimentación ha generado un hecho singular, detectable en la industria editorial de todos los países: el incremento generalizado de los textos impresos, la apuesta por la calidad y el producto bien resuelto y cuidado por parte de la edición más convencional. Perdida la pugna por la funcionalidad, el único, y el mejor espacio, en el que la edición convencional puede competir con la digital, es en el de lo estrictamente bibliográfico, en el refinamiento de las prestaciones más inherentemente unidas a lo impreso, las relacionadas con la legibilidad, con el conjunto de convenciones ortotipográficas que convierten a una obra en ágilmente perceptible interior y exteriormente. Esta pugna por la calidad ha verificado lo que señala Bredehoft (2014), esto es, que para leer debemos de ser cada vez más conscientes de nuestra experiencia visual del texto. La materialidad del libro se ha visto reforzada como antinomia de referencia en un entorno cada vez más digital, configurando colecciones y proyectos editoriales cuyas señas de identidad radican en la calidad formal y conceptual. 


\section{LAS NUEVAS MATERIALIDADES}

Schneps (2015) sostiene que nuestra manera de leer ha estado condicionada por las limitaciones tecnológicas establecidas por los formatos físicos, limitaciones que la tecnología y las nuevas prácticas de lectura estarían transformando. De ahí que Soccavo (2015) proponga el concepto de ciberlector para dar fe del paso del homo sapiens al lector aumentado. Para este autor, la disyuntiva no es ya la de lo impreso y lo digital, sino la de constatación de una realidad emergente expresada en formas de percepción de la realidad que transcienden la obra a través de diversas formas de viaje facilitados por las tecnologías emergentes, como los cascos virtuales, las gafas de realidad aumentada, etc.

Toda esta reflexión nos retrotrae a la necesidad de reformular los planteamientos acerca de la materialidad de los textos, revisitar las hipótesis que formularon Roger Chartier y Roger Laufer, pero también Henri-Jean Martin en algunos de sus trabajos, el neozelandés McKenzie y otros estudiosos que comenzaron a plantear la no neutralidad de los soportes.

Señalaba Laufer (Varry 2012) cómo las técnicas de producción material influencian los productos del espíritu que son los textos. Esta materialidad no es un simple ruido que es necesario filtrar para acceder al mensaje, sino una parte inherente de éste en sí mismo. "No existe texto sin soporte ni libro verdaderamente blanco sino a la espera de un texto", señala este autor. Es necesaria, señala, la creación de una semiología histórica del libro y su objetivo central en el conocimiento del libro y la naturaleza de la comunicación impresa. Conocimiento que "no toma su pleno sentido más que en el estudio más general de la comunicación social escrita y oral, gráfica y audiovisual, ayer, y hoy, en otros lugares y aquí mismo".

Este mismo sentido tienen las formulaciones de Roger Chartier quien manifiesta que " en contra de una definición estrictamente semántica del texto... hay que insistir en que las formas producen sentido, $y$ en que un texto, estable en sí mismo, puede verse investido de un significado y de una tipología inéditos cuando cambian las estructuras o las intermediaciones que lo proponen a la lectura o la escucha". Esta es la orientación de las formulaciones planteadas en la introducción de la obra de Mckenzie acerca de la bibliografía y la sociología de los textos, en la que subraya el sentido que adquieren las formas en cualquier obra: “ Un texto (...) está siempre inscrito en una materialidad (...). Cada una de sus formas está organizada según unas estructuras propias que juegan un papel esencial en el proceso de producción de sentidos. Para atenerse al impreso, el formato del libro, las disposiciones de la puesta en 
página, las convenciones tipográficas están investidas de una 'función expresiva' y portan la construcción de la significación. Organizados por una intención, la del autor o la del editor, estos dispositivos formales tienden a determinar la recepción, a controlar la interpretación, a cualificar el texto. Estructuran el inconsciente de la lectura o de la escucha y son los soportes del trabajo de interpretación". Tanto la imposición como la apropiación del sentido de un texto son pues dependientes de formas materiales cuyas modalidades y disposición, durante mucho tiempo consideradas insignificantes, delimitan las comprehensiones queridas o posibles. Por su parte, Mckenzie precisa que es necesario estudiar los textos en tanto que "formas conservadas, así como sus procesos de transmisión, de la producción a la recepción".

Estas ideas, referidas a los textos impresos revisten una completa actualidad con respecto a las textualidades digitales, en las cuales se concilian todos los nuevos elementos de la cadena de creación, producción y recepción de una manera sistémica, que exige su análisis desde el punto de vista de los productos, pero también de los procesos. La legibilidad está profundamente vinculada con la materialidad textual, con la identidad textual cada vez más polimorfa y diversificada a través de aplicaciones de todo tipo. Es preciso igualmente acercarse a las diversas retóricas de la materialidad, expresadas en formas que se van enriqueciendo a medida que la tecnología y la innovación van generando discursos complementarios que van produciendo una resignificación de los códigos sociales de apropiación de los contenidos. Los cambios en las materialidades han desencadenado una profunda transformación en la percepción de los contenidos y en el propio concepto de libro (Alonso; Cordón 2015). Considerada como parte de un sistema social más amplio, la publicación se articula más que como un acto de clausura, como un acto de socialización. Por sí mismos, los manuscritos tienen un alcance muy limitado, carecen de las formas y garantías que los hacen consumibles por el público en general. La publicación es el proceso de producir un artefacto público e insertarlo en un determinado circuito social. De hecho, la inteligibilidad general de los manuscritos se debe, no a la autonomía de la información, sino a la comprensión por parte del lector de un sistema literario amplio. Los manuscritos no se leen como una forma más pura de libro sino como versiones incompletas, prototipos rápidos del artefacto en que pretenden convertirse. Darles forma pública exige un trabajo más productivo. Por tanto, lo que desde una concepción aperturista se consideran restricciones materiales de las que el texto debe liberarse, son sobre todo recursos sociales que, si se eliminan, necesitan reconstituirse o invocarse de alguna manera para 
mantener el estatus del texto. Chartier tiene razón al señalar que el significado cambia cuando cambia la forma. MacGann lo denomina "la red entrelazada de códigos lingüísticos y bibliográficos". Los cambios sistémicos van más allá de efectos semióticos particulares, alterando nuestra comprensión, no solo de lo que podrían significar las cosas, sino también de por qué son importantes. Los nuevos sistemas de publicación liberan a los documentos de la "barbarie" aceptada en su proceso de trasmisión (Benjamin 2002), descubren críticamente las conexiones "olvidadas" entre los mismos, para capturar el contexto global y complejo de la edición.

\section{DEL TEXTO LINEAL AL TEXTO EN RED}

La probabilidad de que los factores estructurales (que en un campo en particular se hallan en el principio de una tensión crítica) engendren una situación de crisis, favorable a la aparición de acontecimientos extraordinarios, que el funcionamiento normal torna impensables o, por lo menos, "excepcionales" y "accidentales", y por lo tanto desprovistos de eficacia y de significación sociales, alcanza su máximo cuando se cumple la coincidencia de los efectos de muchas crisis latentes de intensidad máxima. Se responde con ello al modelo de las tecnologías disruptivas, esto es, tecnologías o innovaciones que conducen a la desaparición de productos, servicios que utilizan preferiblemente una estrategia disruptiva frente a una estrategia sostenible, a fin de competir contra una tecnología dominante buscando una progresiva consolidación en un mercado. El sistema de innovaciones discontinuas, propio del modelo al que nos estamos refiriendo, ha alimentado la emergencia y consolidación del mercado del libro electrónico, dando lugar a lo que Filloux y Gallete (Filloux; Gassee 2012) denominan como la Disrupción Gigante. Según estos autores "the eBook will become the publishing market's primary engine. Authors will go digital-first and the most successful will land a traditional book deal with legacy publishers".

Como señala Robert Darnton (2009), toda tentativa para explorar el futuro, afrontando los problemas del presente, debería fundarse sobre el estudio del pasado. Los procesos de cambio tecnológico no afectan únicamente al soporte sino también al discurso, como sugiere Cameron Leckie (2010), a las metáforas que lo alimentan y a su contextualización. Como señalaba Derrida, la desaparición del libro es también el nacimiento del libro y el comienzo de la escritura-lectura. 
Todas las consideraciones que habían rodeado al libro y la lectura hasta finales del siglo XX se habían articulado en torno a una concepción y a un sistema estable en el cual apenas se produjeron alteraciones considerables, de tal manera que podíamos hablar de invarianzas en el marco general evolutivo (Cordón; López 2012). Los cambios comienzan a acelerarse a partir de la segunda mitad de la década de los años 90, cuando el nacimiento de Internet le otorga una dimensión nueva a la función y el hecho de comunicar y se han acelerado en los últimos cinco años pergeñando un fenómeno en el que los géneros se van indiferenciando cada vez más y se va articulando una estructura web cada vez más editorializable. Desde este punto de vista es preciso situar la evolución del libro y de la lectura en el marco general de una teoría de la evolución de las representaciones reguladas, esto es, una representación en la que la producción y el uso están sometidos a un conjunto de reglas que se articulan en dos procesos: la mecanización de las reglas de producción y la mecanización de las reglas de uso. Y es a través de esta doble mecanización como las representaciones acaban convirtiéndose en verdaderos sistemas integrados. Los libros han intentado ofrecer, desde Gutenberg principalmente, una solución para organizar un discurso en el espacio. En este sentido desempeñan una función arquitecturante, en la medida en la que permiten la acogida de narraciones ricas y demostraciones complejas en escenarios materialmente reconocibles y limitados (el espacio de un volumen).

Lo que la edición digital ha permitido es una gestión suplementaria a estos modelos estructurales, que han evolucionado a través de tres vertientes fundamentales, estandarizando bien los formatos, adaptando el contenido según las interfaces de lectura utilizados; bien su estructura semántica, en la que textos e imágenes utilizados se asocian a nudos semánticos bien definidos que permiten una modelización y una acumulación de capital semántico; bien las métricas de uso que permiten trazar los rastros dejados por el lector en el libro, vía notas, subrayados, préstamos, etc. Vertientes que confluyen en la actualidad para configurar el fenómeno definido como editorialización de la web (Cordón García 2014).

\section{LA EDITORIALIZACIÓN DE LA WEB}

Desde comienzos de los años 2000 se había detectado la progresiva interacción entre representación de la información, dispositivos y plataformas, dando lugar a la conceptualización de un fenómeno en el que la integración y la 
interoperabilidad eran sus rasgos característicos. Brigitte Guyot (2004) utiliza el término para referirse tanto a los dispositivos que permiten la mediación entre la información, como al proceso de transferencia de la misma. Por su parte Bachimont (2007) considera que este reviste en conjunto tres características:

- Un vínculo profundo entre la actividad de la edición y la práctica de la producción de contenidos en el ámbito digital.

- Un proceso de fragmentación en la producción digital de contenidos.

- Un proceso de recontextualización nacido de la exigencia de adaptar el contenido no digital a un entorno digital.

Debido al aumento de la actividad editorial digital en los últimos años, el concepto ha tenido un gran éxito y ha sido empleado por numerosos estudiosos y actores en la publicación digital. La finalidad era estudiar el impacto de la tecnología digital en la circulación de conocimientos y en el proceso de edición. Sin embargo, no todo podría ser descrito como edición, al menos no en el sentido tradicional del término. No se puede, por ejemplo, hablar de la publicación digital sin abordar las diferencias entre las prácticas impresas y digitales, y estas diferencias de alguna manera deben de ser abordadas a fondo. Al mismo tiempo, el papel particular de la red de personas detrás de cualquier publicación desborda las nociones convencionales de consejos de publicaciones o editoriales. Por esta razón la editorialización aborda estas diferencias, incidiendo en el conjunto de prácticas que no pueden ser agrupadas en el conjunto de fenómenos que sirven para caracterizar lo que tradicionalmente denominamos como "edición".

De ahí dos cuestiones fundamentales estrechamente relacionadas con esta reapropiación del término. Por una parte, está el hecho de que los contenidos digitales tienen su propia especificidad, de tal manera que los desarrollos tecnológicos condicionan la estructuración del pensamiento y generan su propio ecosistema, como planteara brillantemente Chartier (2005), determinando cambios profundos en el orden de las propiedades, de los discursos y de las razones. Por otra, la constatación de que la fragmentación de los contenidos en el espacio digital establece una relación compleja entre ellos que exige su reordenación en unidades de significado. Cuando se habla de la progresiva indiferenciación de géneros se apela a un conjunto de fenómenos que tiene que ver con el debilitamiento de las fronteras tradicionales entre canales comunicativos, de tal manera que asistimos a un serialización de los libros, que ha sido aprovechado por Thomson Reuter por ejemplo para el desarrollo 
de sus sistema de Book Index, asimilándolo a los índices de citas que ya desarrollaba con las revistas, o de creación de corpus a partir de conjuntos de artículos vinculados temáticamente.

Consideramos entonces la editorialización como el conjunto de dispositivos técnicos (redes, servidores, plataformas, algoritmos de los motores de búsqueda), estructuras (hipertexto, multimedia, metadatos) y prácticas (anotaciones, comentarios, recomendaciones, a través de redes sociales) que permiten producir, organizar y activar la circulación de contenidos en la web (VitaliRosati 2014), garantizando su máxima visibilidad y posibilidades de acceso.

Desde el punto de vista de la editorialización no se puede considerar el entorno digital como un espacio discreto y separado del contexto analógico. Por el contrario, como defiende O'Donnell (2015), es preciso tener en cuenta la fluidez que existe entre el espacio digital y predigital. Además, en una fase de transición como la que nos encontramos las continuidades y discontinuidades entre ambos entornos son fundamentales para comprender el contexto de las fuentes de información en la actualidad. La misma articulación de la lectura en dos modalidades básicas de dispositivos apunta a esta adaptación. De tal manera que los dispositivos dedicados de tinta electrónica responderían a ese movimiento mimético que toda revolución de los medios entraña, mediante el cual se intenta trasladar las características del sistema antiguo al nuevo modelo para desencadenar una transición no traumática. Los primeros pasos de lo digital, con el diseño de estanterías, cubiertas de los libros idénticas a las de papel, funciones de representación completamente idénticas, eran deudores de esta concepción continuista que, en cierto modo, constituye una forma de resistencia a la constitución de un espacio autónomo y diferenciado.

Como señala Vitali-Rosati (2014) la editorialización implica la presencia de una serie de atributos que son connaturales a ella. En primer lugar, podemos hablar de que se trata de un proceso, y más concretamente de un proceso abierto, en movimiento, dinámico. El carácter procesal implica que es muy difícil de identificar y aislar un acto con significación autónoma separado del resto. Cada actividad está de alguna manera relacionada con las demás, y es imposible delimitar categóricamente una cadena precisa de acciones. Consideremos el ejemplo de la publicación de un artículo científico. La publicación de un artículo académico en una revista impresa es un proceso cuyos componentes pueden ser definidos y aislados: hay un autor que escribe el artículo, un consejo científico y otro editorial de la revista, dos o tres revisores para cada una de las contribuciones, y finalmente un grupo de personas que desempeña el trabajo más técnico relacionado con la publicación de la revista. 
Estos eslabones de la cadena de publicación se pueden contar: puede haber más o menos, pero el número será en cualquier caso preciso. El proceso editorial comienza cuando se propone el documento para su publicación por parte del autor, y termina cuando la revista se imprime y se distribuye. Una vez que la revista se imprime, el papel es algo estable y estático. Se podría argumentar que la distribución de la revista, las reacciones de sus lectores y el número de citas que recibe es importante y debe ser considerado como parte del proceso editorial. Aunque esto es cierto, también es innegable que el momento crucial de la impresión significa una discontinuidad en el proceso: el documento se mantendrá tal y como aparece en ese momento. El grupo específico de personas que trabajan en la publicación sigue el proceso desde su inicio, cuando el autor propone el documento, hasta el final (o al menos un extremo), la impresión.

En el caso de un documento en línea, la identificación de su publicación como un proceso aislado y definido es más compleja. A diferencia del proceso impreso, una vez que el artículo ha sido aprobado, maquetado y producido, no acaba el proceso. Primero, el lugar que ocupa el artículo no está decidido. En una revista impresa, el editor decidiría dónde aparecerá el documento: en qué posición en la revista, en qué páginas, si va a figurar alguna información relativa al mismo en la cubierta o no. Para un documento en línea, su posición -y en última instancia su significado-depende de un conjunto de factores que son externos al grupo de redacción: la posición que ocupará en la lista de resultados de búsqueda, por ejemplo. Si, en la publicación impresa, las relaciones entre los diferentes trabajos son propuestas por el editor-que ha puesto a propósito determinado material junto en la misma revista impresaen el caso de un documento en línea, las relaciones también se generan por otras plataformas, que agregan el contenido y lo pueden viralizar en contextos muy diferentes, que pueden ir desde redes sociales como Twitter, a sistemas de referencia social como Research Gate, Mendeley, etc. Por supuesto, la misma estructura de difusión y comentarios pueden darse en una revista impresa, pero en el entorno digital estas estructuras no constituyen un espacio separado, sino inherente a la propia naturaleza de entorno digital. Además, la vida del artículo continúa tras la finalización del trabajo de los editores, y estos carecen de capacidad de control alguna sobre el mismo. La revista, por lo tanto, ya no es una forma estable de circulación, debido a que el acceso a un documento no depende exclusivamente de ella, sino más bien de otras plataformas, como un motor de búsqueda o una red social; y el documento se convierte en un objeto inestable que puede ser fragmentado: se 
pueden extraer datos de un documento y considerar los datos como unidades originales que, a su vez, pueden combinarse de muchas maneras diferentes (Stern, Guédon, Jensen 2015). El elemento procesal que caracteriza a la cultura digital no es un fenómeno totalmente nuevo, pero, aun existiendo en la cultura impresa, está mucho menos desarrollado. Los cambios históricos no deben ser interpretados como rupturas epistemológicas sino más bien como la reorganización de los principios culturales preexistentes. Lo digital revoluciona las relaciones entre la materialidad del objeto y la identidad del texto, así como entre materialidad y formas de circulación, todas ellas sujetas a un entramado de movilidades múltiples, en el que los elementos procedimentales se van haciendo cada vez más ricos.

Un claro ejemplo de esto lo constituye la noción de autor, o de autoridad como instancia de legitimación del contenido. Frente al modelo impreso, en que esta recae fundamentalmente sobre la revista o el libro y el autor, ahora se multiplica en virtud de la red de relaciones que se establecen en torno al texto. En un entorno digital, de naturaleza dinámica, el autor, de carácter estático, es una autoridad entre otras, que confiere legitimidad a un contenido. El editor está en la misma situación: el editor puede ser una autoridad que legitima un documento solo si éste puede ser entendido en un sentido definido y estable. Sin embargo, un documento no puede considerarse un todo independiente y coherente, en la medida en que no puede establecerse con precisión cuando comienza y termina el proceso de su producción, siendo aún más difícil de determinar el conjunto de instancias involucradas en su creación y la circulación.

El concepto de autoridad se diluye de manera reticular mediante la distribución de diferentes formas de responsabilidad según el conjunto de las intervenciones. Realmente asistimos no tanto a la legitimación de un objeto, un artículo, un libro, etc., sino de un proceso, esto es, el conjunto de discursos que se articulan en torno a ellos que, en muchos casos, pueden determinar intercambios sustanciosos que pueden verse abocados a cambios de los contenidos originales. Se produce por lo tanto un desplazamiento de la autoridad desde los elementos de la cadena de valor de la comunicación al proceso en su conjunto.

Lo importante, entonces, no es la unidad de un texto producido por una persona -y legitimado por él / ella, o por un editor- sino la colección de relaciones dinámicas que este contenido mantiene con otros. Es el conjunto de relaciones y vínculos las que hacen que el contenido sea accesible y visible. Las acciones de lectura y escritura se fusionan en un proceso de editorialización 
general y la autoridad se articula entre la distribución de conexiones que constituyen el espacio web. Debido a que el proceso permanece abierto, sin embargo, la autoridad no puede legitimar un objeto: tiene que legitimar un proceso. De modo que podríamos hablar del desplazamiento de la autoridad como objeto a la autoridad como proceso.

En el entorno digital la autoridad está estrechamente relacionada con la actividad, constituyendo esta otra de las características de la editorialización. Cuando más actividad se despliega en la red, mayor reputación virtual se detenta y mayor autoridad se confiere al responsable de las intervenciones. Este fenómeno ha dado lugar a que un elemento de carácter cuantitativo, como el número de intervenciones, de citas, de descargas, de visualizaciones, se transforme en un factor de dimensión cualitativa representada por un capital simbólico que se acumula mediante mayor reputación y prestigio, y un capital académico, cada vez más deudor de estos procesos de reconocimiento y acreditación.

Conceptos como los Inteligencia colectiva (Levy 2007) o Inteligencia conectada (Giglio; Baldessar 2012), son inherentes a una forma de comunicación en la que la vocación de mensaje totalizado y universal, propia de la ilustración y del soporte impreso, se diluye en una pluralidad posibilitada por la interactividad y la estructura de campo del entorno digital. Como señala Pierre Levy "es difícil entender un mensaje cuando se lo separa de su contexto vivo de producción". Esta es la razón por la que apareciesen, del lado de la recepción toda una tecnología lingüística de apoyo, y del de la emisión un esfuerzo por componer mensajes que puedan circular por todas partes, independientemente de sus condiciones de producción. Mensajes que, en la medida de lo posible, han de llevar sus claves de interpretación. Lo que se ha roto es la pretensión de clausura semántica inherente al ámbito de lo impreso, favoreciendo formas discursivas nuevas emparentadas con las reapropiaciones y reinterpretaciones permanentes que forman parte constitutiva del proceso de producción de contenidos. En el texto de Fedro platónico, señala Lledó (2014), hay una importante indicación en la que se encierra el germen de la teoría de la interpretación, o sea de aquella mirada sobre lo escrito que pretende descubrir su sentido desvelando, en él, la mayor parte de sus ocultas o diluidas referencias. "a ti tal vez te importa quién es y de donde es el que habla”. En estas dos interrogaciones ¿quién es? y ¿de dónde? se comprime la posterior teoría de la hermenéutica. El quién del autor y el dónde de las circunstancias históricas que le condicionan fueron las claves sobre las que levantaba ese complejo edificio que denominamos contexto 
en el ámbito impreso. Esos elementos fundacionales de la creación y de la recepción ahora adoptan disposiciones reticulares y colectivas, de forma que lo que da sentido a los contenidos es, como señala Levy, su vinculación con una comunidad activa.

De cualquier modo, el concepto de autoridad no deja de ser una dimensión inestable y cambiante, tanto desde el punto de vista científico como social. En una investigación llevada a cabo durante el año 2015, y publicada en febrero de 2016, sobre la transmisión de la cultura en la era digital, se interrogaba, entre otras muchas cosas, sobre las figuras que mejor encarnaban los valores de la cultura. El resultado fue el siguiente:

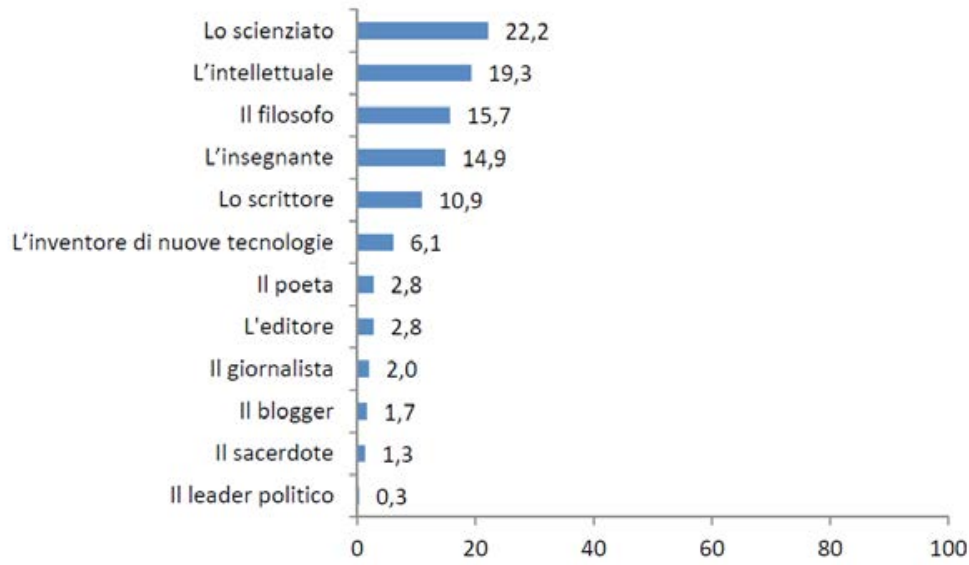

Fig. 3. Transmisión de la cultura en la era digital

Fuente: La trasmissione della cultura nell'era digitale: Una inchiesta sul sapere (2016)

Independientemente de la posible yuxtaposición semántica de conceptos, cuya dimensión anfibológica podría restar consistencia a la indagación (¿el filósofo no es un intelectual, y el escritor?, etc.) lo interesante estriba en la asignación de pesos a las diferentes figuras, y la aparición de otras en una nómina que podría ser fácilmente ampliable, como muestra de la diversificación de las representaciones contemporáneas de la legitimación cultural. 


\section{CONCLUSIONES}

Gutenberg consiguió desarrollar una de las metáforas más poderosas de las edades moderna y contemporánea, la del libro, cuyo entorno y formas de consumo constituyen una de las hierofanías más inmersivas existentes. Con la experiencia digital la hierofanía va derivando hacia la heterofanía, en el sentido de que gran parte del poder metafórico de ese entorno se va diluyendo por la paulatina desaparición de sus hitos constituyentes: el libro como resumen del mundo, como recipiente donde este entra, se va transformando en un objeto de puente hacia él. La lógica inductiva se transforma en lógica difusa, el orden del libro, encarnado en el cartesianismo racionalista, va derivando hacia otra metáfora, la del laberinto. Si la edición ha sido el camino de la legibilidad y la lecturabilidad, a través de convenciones tipográficas de distinto signo, la web recorre cada vez con más frecuencia el mismo. Esta normalización implícita de los sistemas de lectura, que por otra parte se hacen más abiertos e inconsistentes semánticamente, debido a su carácter fragmentario, conectado y nómada, afecta a otro fenómeno cada vez más importante: el de la documentalización de los libros. La web se ensimisma, reforzando los elementos conceptuales y formales propios de la tipografía 2.0. Frente a la externalización de los enlaces e hipervínculos se potencian las inferencias de la intervención del usuario. Si la espacialidad y fisicidad implicaban asociaciones mentales de recuperabilidad implícitas en el ámbito de lo impreso, la reproducción de los esquemas estructurales del mismo, mediante transformaciones automáticas, gracias a software de intervención débil como Magic Scroll, DotDotDot, Readability, etc., están dando lugar a experiencias que pueden resolver algunos de los inconvenientes analizados más arriba. Todo esto nos sitúa en un contexto en el que la mediación documental va a estar, o ya está, profundamente determinada por la porosidad cada vez mayor entre el mundo "real" y los nuevos territorios digitales. Las nuevas interfaces de realidad aumentada, el Internet de las Cosas, el progreso en el campo de la inteligencia artificial, etc. están rediseñando los conceptos de lectura y de lector inherentes a una tradición vinculada con la imprenta. No es extraño por lo tanto el nacimiento de cierta melancolía ante el desarrollo de propuestas como las de Moretti (2013), que con su concepto de Distant Reading apela a una práctica de la lectura hiperconectada, vinculada con el movimiento de los big data, en la que la obra individual carece de significado, y es el entramado y la intersección lo que confiere visibilidad y proyección a cientos de obras tratados con algoritmos semánticos. Lo que Piper (2012) 
denomina "lectura topológica", aquella que busca patrones y modelos, vectores de uso, en miles de documentos, es la consecuencia de una mecanización en los sistemas de producción y consumo favorecida tanto por la tecnología como por el desarrollo de prácticas cada vez más reguladas en el uso de esta.

Lo digital reviste por lo tanto una dimensión ambivalente. Por una parte, potencia la visibilidad y accesibilidad a través de múltiples plataformas y dispositivos, permite la integración del lector en un proceso en el que siempre había quedado marginado y propicia la participación colaborativa en todo tipo de escritos. Pero por otro, genera discursos regidos por modelos cada vez más distanciados de ese concepto sagrado y casi litúrgico de la lectura, cuyos orígenes se remontan a Agustín de Hipona (Wellmon 2015), en los que la aproximación individual, ensimismada y concentrada constituye las señas de identidad del lector. Piper (2013) lo denomina "la nostalgia de la lectura bibliográfica", de la obra individual como suma y epitome de adquisición del saber. Pero más allá de lo individual subyace lo que Daston (2014) califica como "la profunda unidad que vincula lo aparentemente misceláneo", y que esta vertiente de lo digital está desvelando a través de protocolos de análisis sumamente interesantes para aflorar estructuras ocultas en el bosque de los demasiados libros. Estas innovaciones no atentan contra la pervivencia de los libros, en palabras de Simon Eliot, Jonathan Rose (2009), supervivientes que durante más de cinco mil años de historia se han movido de una forma material a otra, adaptándose a todo tipo de culturas, reinventándose continuamente para ofrecer nuevas formas y nuevos servicios.

\section{BIBLIOGRAFÍA}

Alonso Arévalo, Julio; Cordón García, José Antonio. El libro como sistema: Hacia un nuevo concepto de libro. Cuadernos De Documentación Multimedia, 2015. http://revistas.ucm. es/index.php/CDMU/article/view/50628

Arguelles, Juan Domingo. Si quieres... lee. Contra la obligación de leer y otras utopías lectoras. Forcola, 2009.

Bachimont, Bruno. "Nouvelles tendances applicatives: de l'indexation à l'éditorialisation". L'indexation multimédia, Paris: Hermès, 2007.

Benjamin, Walter. La dialéctica en suspenso. Fragmentos sobre la Historia. Arcis-Loom, 2002 Bourdieu, Pierre. Las reglas del arte. Barcelona: Anagrama, 2011.

Bredehoft, Thomas A. The Visible Text: Textual Production and Reproduction from Beowulf to Maus. Oxford University Press, 2014. 
Burke, Peter. Los avatares de El Cortesano: lecturas y lectores de un texto clave del espíritu renacentista. Barcelona: Gedisa, 2009.

Chartier, Roger. Lenguas y lecturas en el mundo digital. El presente del pasado: escritura de la historia, historia de lo escrito. Iberoamericana, 2005, pp. 195-220.

Comercio Interior del libro 2015. Madrid: Federación de Gremios de Editores, 2016.

Cordón García, José Antonio. Editorialización de la Web y nuevas mediaciones en el entorno de la lectura digital. En María Teresa Fernández Bajón et al. Nuevos hábitos de consumo de información y lectura para la inclusión social. Madrid: Universidad Complutense, 2014, pp. 37-54.

Cordón-Garcia, José-Antonio y Carlos-Alberto Lopes. "El libro electrónico: invarianzas y transformaciones". El profesional de la información, 2012, v. 21, n. 1, pp. 83-90.

Cordón García, J. A., J. Alonso Arévalo y R. Gómez Díaz. Social reading: Platforms, aplications, clouds and tags. Chandos Publishing, 2013.

Darnton, Robert. "The Case for Books: Past, Present, and Future”. PublicAffairs, 2009.

Daston, Lorraine. "Wonder and the Ends of Inquiry". The Point 8, 2014, pp. 105-11, http:// thepointmag.com/2014/examined-life/wonder-ends-inquiry.

Eliot, Simon y Jonathan Rose. A Companion to the History of the Book. Blackwell, 2009.

Filloux, F. y J. L. Gassee. "Ebooks: the giant disruption". Monday Note, 2012. http://www. mondaynote.com/2012/02/26/ebooks-the-giant-disruption/

Finnegan, Ruth. Why Do We Quote?: The Culture and History of Quotation. Open Book Publishers, 2013.

Giglio, K. y M. J. Baldessar. Cibercultura y convergencia: contribuciones de Kerckhove. Editorial Académica, 2012.

Guyot, Brigitte. Sciences de l'information et activité professionnelle, vol.38, C.N.R.S. Editions, 2004.

Iser, Wolfgang. Stepping Forward: Essays, Lectures and Interviews. Crescent Moon Publishing, 2008.

Leckie, Cameron. The abandonment of technology. http://www.energybulletin.net/ stories/2010-10-16/abandonment-technology, 2010.

Levy, Pierre. Cibercultura: la cultura de la sociedad digital. Anthropos, 2007.

Lledó, Emilio. Fedro. Madrid: Gredos, 2014.

Michael, Anne. What is The Role of Social Media in Scholarly Publishing? Scholarly Kitchen, 2016. https://scholarlykitchen.sspnet.org/2016/07/28/ask-the-chefs-what-is-the-role-ofsocial-media-in-scholarly-publishing/

Moretti, Franco. Distant Reading. Verso, 2013.

O’Donnell, Daniel Paul (2015). A “Thought Piece" on Digital Space as Simulation and the Loss of the Original, [http://dpod.kakelbont.ca/2015/02/11/a-thought-piece-on-digitalspace-as-simulation-and-the-loss-of-the-original/].

Panorámica de la Edición Española de Libros 2015. Madrid, Ministerio de Educación y Cultura, 2016.

Piper, Andrew. Book Was There Reading in Electronic Times. University Chicago Press, 2012. 
Reading's Refrain: from Bibliography to Topology, ELH 80, 2013, pp. 373-99. http://piperlab.mcgill.ca/pdfs/Piper_ReadingsRefrain.pdf

Stern, Niels; Jean-Claude Guédon \& Thomas Wiben Jensen. "Crystals of Knowledge Production. An Intercontinental Conversation about Open Science and the Humanities", Nordic Perspectives on Open Science 2015 (1), http://dx.doi.org/10.7557/11.3619

Sinclair, S. \& G. Rockwell. Les potentialités du texte numérique. In M. E. Sinatra, \& M. VitaliRosati (Eds.), Pratiques de l'édition numérique (pp. 191-204). Presses de l’Université de Montréal, 2014.

Schneps, H. Using technology to break the speed barrier of reading. Scientific American, 2015. http://www.scientificamerican.com/article/using-technology-to-break-the-speedbarrier-of-reading

Soccavo, L. ¿La disparition du livre est-elle inevitable? Viavooks, 2015

Varry, D. Qu'est-ce que la bibliographie matérielle? Enssib, 2012

Vitali-Rosati, Marcello. Digital Paratext. Editorialization and the very death of the author. Examining Paratextual Theory and its Applications in Digital Culture, IGI Global, Nadine Desrochers and Daniel Apollon, 2014, p. 110-127.

Wellmon, Chad. Sacred Reading: From Augustine to the Digital Humanists. The Hedgehog review: vol. 17 no. 3 (fall 2015). http://www.iasc-culture.org/THR/THR_article_2015 Fall_Wellmon.php 